\title{
The Transcription Regulator Patz1 Is Essential for Neural Stem Cell Maintenance and Proliferation
}

\author{
Sara Mancinelli ${ }^{1,2,3}$, Michela Vitiello ${ }^{4}$, Maria Donnini ${ }^{1}$, Francesca Mantile', \\ Giuseppe Palma ${ }^{5}$, Antonio Luciano ${ }^{5}$, Claudio Arra ${ }^{5 \neq}$, Laura Cerchia ${ }^{4}$, \\ Giovanna Lucia Liguori't+ and Monica Fedele ${ }^{4 * t}$
}

${ }^{1}$ Institute of Genetics and Biophysics, National Research Council, Naples, Italy, ${ }^{2}$ Department of Biomedical Sciences, Humanitas University, Pieve Emanuele, Italy, ${ }^{3}$ Istituto di Ricovero e Cura a Carattere Scientifico (IRCCS) Humanitas Research Hospital, Rozzano, Italy, ${ }^{4}$ Institute for Experimental Endocrinology and Oncology, National Research Council, Naples, Italy, ${ }^{5}$ Struttura Semplice Dipartimentale (S.S.D.) Sperimentazione Animale, Istituto Nazionale Tumori-Istituto di Ricovero e Cura a Carattere Scientifico (IRCCS)-Fondazione G. Pascale, Naples, Italy

OPEN ACCESS

Edited by: Carmen Castro,

University of Cádiz, Spain

Reviewed by:

Elena Parmigiani,

University of Basel, Switzerland

Angela Fontan-Lozano,

Seville University, Spain

*Correspondence: Monica Fedele mfedele@unina.it;

monica.fedele@cnr.it

tThese authors share last authorship

¥Present address: Claudio Arra,

Retired, Naples, Italy

Specialty section:

This article was submitted to

Stem Cell Research,

a section of the journal

Frontiers in Cell and Developmental

Biology

Received: 22 January 2021

Accepted: 15 March 2021

Published: 07 April 2021

Citation:

Mancinelli S, Vitiello M, Donnini M, Mantile F, Palma G, Luciano A, Arra C,

Cerchia L, Liguori GL and Fedele M

(2021) The Transcription Regulator Patz1 Is Essential for Neural Stem Cell

Maintenance and Proliferation. Front. Cell Dev. Biol. 9:657149. doi: 10.3389/fcell.2021.657149
Proper regulation of neurogenesis, the process by which new neurons are generated from neural stem and progenitor cells (NS/PCs), is essential for embryonic brain development and adult brain function. The transcription regulator Patz1 is ubiquitously expressed in early mouse embryos and has a key role in embryonic stem cell maintenance. At later stages, the detection of Patz 1 expression mainly in the developing brain suggests a specific involvement of Patz1 in neurogenesis. To address this point, we first got insights in Patz1 expression profile in different brain territories at both embryonic and postnatal stages, evidencing a general decreasing trend with respect to time. Then, we performed in vivo and ex vivo analysis of Patz1-knockout mice, focusing on the ventricular and subventricular zone, where we confirmed Patz1 enrichment through the analysis of public RNA-seq datasets. Both embryos and adults showed a significant reduction in the number of Patz1-null NS/PCs, as well as of their selfrenewal capability, compared to controls. Consistently, molecular analysis revealed the downregulation of stemness markers in NS/PCs derived from Patz1-null mice. Overall, these data demonstrate the requirement of Patz1 for NS/PC maintenance and proliferation, suggesting new roles for this key transcription factor specifically in brain development and plasticity, with possible implications for neurodegenerative disorders and glial brain tumors.

Keywords: neurogenesis, neural stem cells, PATZ1, knockout mice, neurosphere assay, subventricular zone

\section{INTRODUCTION}

The Poxviruses and Zinc finger (POZ)/Broad complex, Tramtrack, and Bric à brac (BTB), and AT-hook containing Zinc finger protein 1 (PATZ1), also referred as MAZ related factor (MAZR), Zinc finger Sarcoma gene (ZSG) or Zinc finger factor/protein (ZNF278/Zfp278), is a transcriptional regulator that modulates the expression of several genes, either negatively or positively depending on the cellular context (Fedele et al., 2000, 2017; Valentino et al., 2013a). PATZ1 belongs to the $\mathrm{POZ}$ and Krüppel (POK) family of transcription factors, which are implicated in many biological processes, including B cell fate determination, cell cycle progression, DNA damage responses and 
development (gastrulation, limb formation, hematopoietic stem cell fate determination) (Kelly and Daniel, 2006; Costoya, 2007). Accordingly, Patz1 has been implicated in T cell differentiation (Ellmeier, 2015; Andersen et al., 2019; Orola et al., 2019), cell cycle regulation (Valentino et al., 2013b), DNA damage response (Keskin et al., 2015), and embryonic development (Valentino et al., 2013b). Noteworthy, a critical role for Patz1 has been described in the maintenance of embryonic stemness through its functional interaction with the pluripotency master genes Nanog and Pou5f1, which are both targets and regulators of Patz1 (Ow et al., 2014). Consistently, mouse embryonic fibroblasts lacking Patzl show low reprogramming efficiency in induced pluripotent stem cells (iPSCs) (Ma et al., 2014).

In situ hybridization analysis demonstrated that Patzl gene is actively expressed during early embryogenesis in several districts including the central nervous system, where it mainly localizes in proliferating neural progenitors (NPCs) of the periventricular neocortical neuroepithelium, suggesting a role for Patz1 in neurogenesis (Valentino et al., 2013b). Indeed, the ventricular and subventricular zone (VZ-SVZ) is located in the wall of the lateral brain ventricles and produces new neurons in both embryonic and postnatal brain of mammals. In the embryonic brain, immature, and neurogenic transient-amplifying NPCs, the intermediate progenitor cells (IPCs), originated from multipotent radial glia cells (RGCs), also known as apical progenitors (APs) in the VZ-SVZ (Gaspard et al., 2008; Okamoto et al., 2016; Jabaudon, 2017; Telley et al., 2019). The IPCs produce a huge variety of neuronal cell types that migrate toward their final destinations, thus contributing to brain formation and function (Mancinelli and Lodato, 2018). In the adult, neural stem/progenitor cells (NS/PCs) are also present in the SVZ. NSCs activate, upon an injury or inflammatory stimulus, to produce transient-amplifying neural progenitor cells (NPCs) that divide and generate a different type of proliferating progenitors, the neuroblasts (NBs), that eventually differentiate to produce newborn neurons (Alvarez-Buylla and Garcia-Verdugo, 2002; Chaker et al., 2016; Belenguer et al., 2021).

Genetic inactivation of Patz1 gene in mice results in a partial embryonic lethality and several developmental defects in testes, cardiac outflow tract and brain (Fedele et al., 2008; Valentino et al., 2013b). The latter defects consist in hypoproliferation of the periventricular tissue, one of the key neurogenic sites, consistently with the idea that Patzl could play a crucial role in neurogenesis (Valentino et al., 2013b).

To verify this hypothesis, we first deeply examined Patz1 gene expression in different sub-regions of the embryonic, fetal, juvenile and adult mouse brain, showing a decreasing trend with respect to time, as opposed to a stable expression of Patz1 at neurogenic sites of the adult brain. Then, we analyzed the in vivo incorporation of the DNA intercalant $5^{\prime}$-bromo- $2^{\prime}$-deoxyuridine (BrdU) at embryonic stage, and we isolated and ex vivo cultured both embryonic and adult NS/PCs from Patz1-null homozygotes, heterozygotes and wild type mice to perform neurosphere assay and gene expression analysis. All together the results indicate that Patzl inactivation leads to a drastic impairment in the number and self-renewal capacity of NS/PCs, therefore pointing out to a key role of Patz1 in maintenance and proliferation of NS/PCs.

\section{MATERIALS AND METHODS}

\section{Analysis of Patz1 Transcript Distribution From Publicly Available Datasets}

To analyze Patz1 expression levels across development in different cellular populations we took advantage of different publicly available datasets. Normalized fragments per kilobase of exon model per million reads mapped (FPKM) expression values were downloaded from "Mouse RNA-seq time-series of the development of seven major organs" experiment of EMBL-EBI Expression Atlas (EMBL-EBI Expression Atlas, 2020; Papatheodorou et al., 2020). The experiment comprises gene expression data (mRNAseq) from different tissues (among them heart, brain, liver, testis, ovary) across several time points from embryonic day 10.5 (E10.5) to postnatal day 63 (P63). Expression data relative to Patz1 in this dataset are represented in the heatmap (A) and in the barplot (B) in Figure 1. Two single cell RNA-seq dataset were also analyzed (C2020 10× Genomics 10K E18 Brain cells; Telley et al., 2019- GSE118953). We downloaded row counts and metadata relative to 10,000 cells from E18 mouse brain from the $10 \mathrm{X}$ Chromium website and data relative to the Telley dataset using GSE118953 accession number in the GEO database ${ }^{1}$. Clustering analysis was performed with R version 4.0 .3 (2020-10-10) using Seurat package version 3.2.3 (Satija et al., 2015; Butler et al., 2018; Stuart et al., 2019). The dataset was normalized using standard SCT normalization pipeline ${ }^{2}$. A cell cycle score was also assigned to each single cell using the Seurat pipeline but was not used as variable to regress during normalization because considered biologically relevant. We considered the top 60 statistically significant principal components as input for UMAP dimensional reduction, using the DimPlot function. To identify cellular clusters, we used FindClusters function considering 0.2 resolution for both datasets. The robustness of the clustering was validated using an in silico downsampling approach. To annotate cell clusters, top 20 differentially expressed genes (markers) per cluster were considered and the most relevant were plotted using DotPlot function to check cell type specificity. Data relative to $10 \mathrm{X}$-Chromium dataset were shown in Figures 2A-D, whereas data relative to the dataset of Telley et al. (2019) were shown in Figures 3A-C. We also analyzed bulk-RNAseq data derived from purified NS/PCs from adult murine SVZ (Belenguer et al., 2021). The normalized expression matrix of the dataset of Belenguer et al. (2021) was downloaded from GEO database using GSE138243 accession number. Expression data relative to Patz1, Cd9, Slc1a3, Tbr2, $D c x a$, and $C d 24 a$ genes were reported in the heatmap (scaled data) and in the floating box plot (normalized expression) in Figures 2E,F.

\section{Animals}

Patz1-knockout mice were previously described (Valentino et al., 2013b). C57/BL6 wild type mice were used to collect

\footnotetext{
${ }^{1}$ https://www.ncbi.nlm.nih.gov/geo/query/acc.cgi?acc=GSE118953

${ }^{2}$ https://github.com/hbctraining/scRNA-seq
} 
A

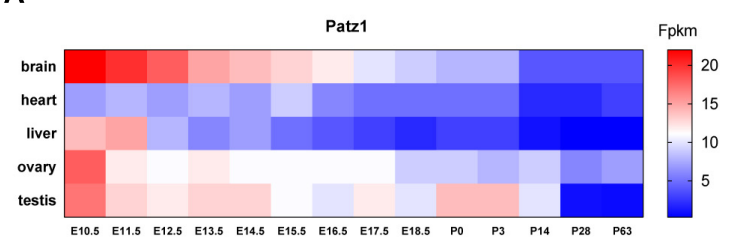

Developmental stage

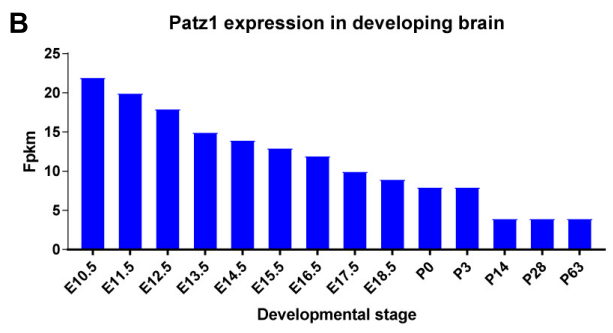

C

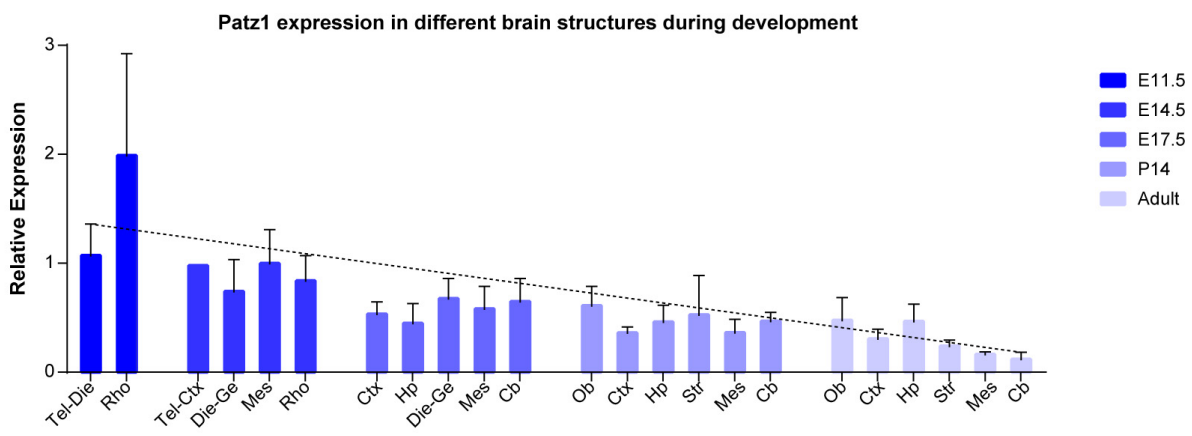

D

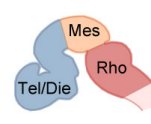

E11.5

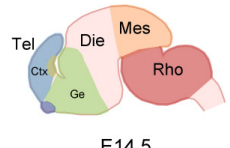

E14.5

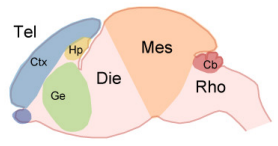

E17.5

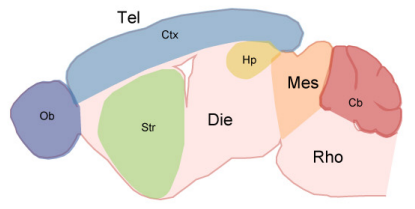

P14-Adult

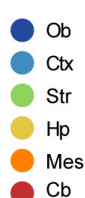

$\mathrm{Cb}$

E
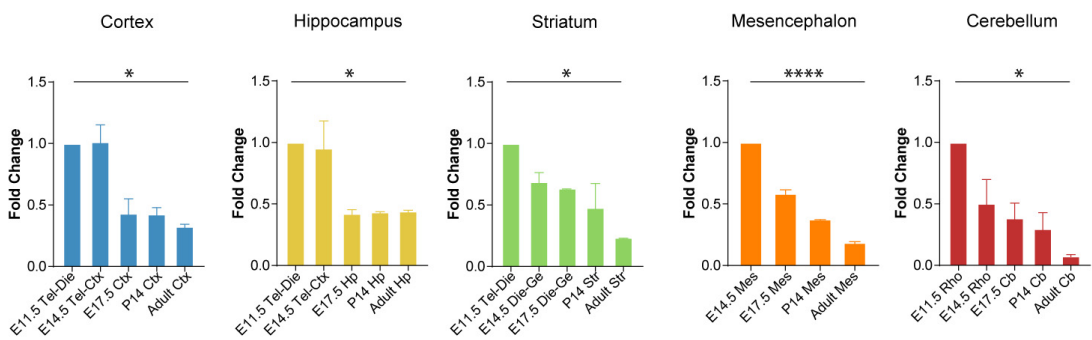

FIGURE 1 | Patz1 expression in embryonic and postnatal tissues and brain structures. (A) Heatmap showing Patz1 expression from EMBL-EBI Expression Atlas in the murine brain, heart, liver, testis, and ovary across development. (B) Bar plot highlighting Patz1 expression levels in the brain during development from EMBL-EBI Expression Atlas. (C) Bar plot showing the relative expression of Patz1 $\left(2^{-\Delta \Delta C t}\right)$ in different embryonic, postnatal and adult brain regions, using the E14.5 Tel-Die sample as calibrator. (D) Schematic representation of brain structures at different developmental stages. (E) Bar plots showing Patz1 fold change expression relative to E11.5 or E14.5 control sample (2- $\left.2^{-\Delta C t}\right)$ in the different microdissected brain structures. Tel, Telencephalon; Die, Diencephalon; Mes, Mesencephalon; Rho, Rhombencephalon; Ob, Olfactory bulbs; Ctx; Cerebral Cortex; Hp, Hippocampus; Ge, Ganglionic eminence; Cb, Cerebellum; Str, Striatum. Graphs are represented as mean values $\pm \mathrm{SD}$ and all the significant differences are indicated by asterisks. ${ }^{*} p<0.05 ; * * * *<0.0001$, as assessed by ANOVA. n.s. $=$ not significant.

mouse brains at different embryonic and post-natal stages of development. Mice were maintained under standardized nonbarrier conditions in the Laboratory Animal Facility of Istituto dei Tumori di Napoli (Patz1-knockout mice and their wild type controls) and in the Animal Facility of the Institute of Genetics and Biophysics (C57/BL6 wild type mice showed in Figure 1) in Naples, Italy. All studies were conducted in accordance with the Italian regulations for experimentations on animals (prot. no. 576/10 approved by the Italian Ministry of Health on 3 February 2011).

\section{Patz1 Genotype Analysis}

Tail pieces from both embryos and adult mice were lysed in tail digestion buffer $(50 \mathrm{mM}$ Tris, $\mathrm{pH} 8.0 ; 100 \mathrm{mM}$ EDTA, pH 8.0; $100 \mathrm{mM} \mathrm{NaCl}$; 1\% SDS) plus proteinase $\mathrm{K}$ $(0.6 \mathrm{mg} / \mathrm{ml})$ over-night at $55^{\circ} \mathrm{C}$. Genomic DNA was extracted by 

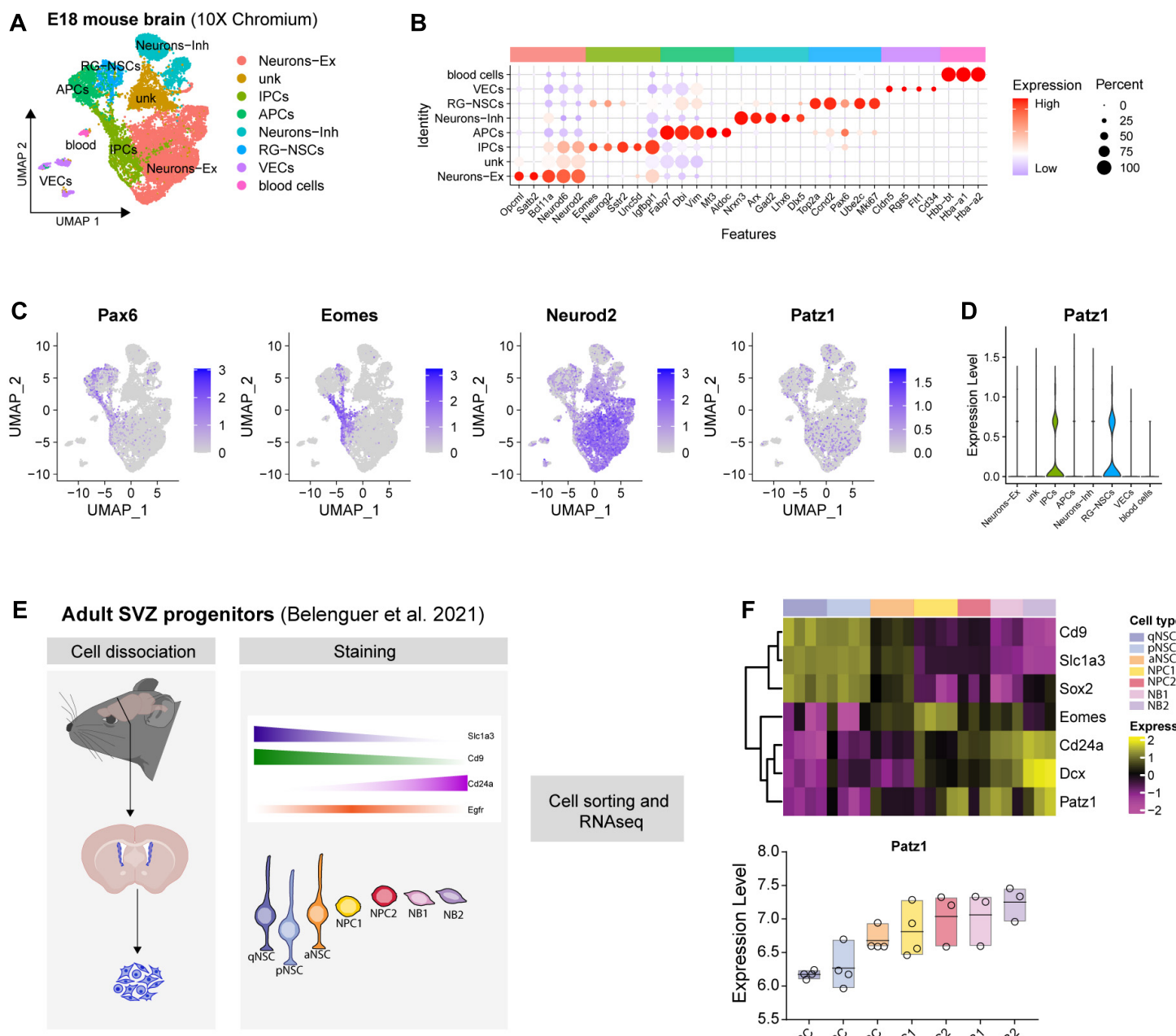

$\mathbf{F}$
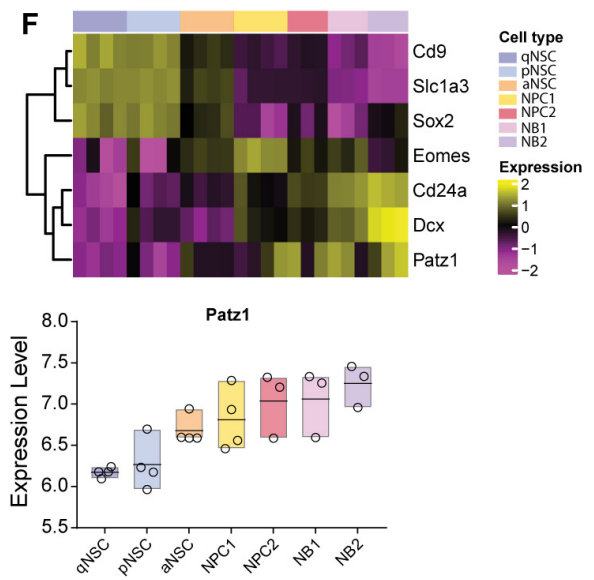

FIGURE 2 | Patz1 expression in embryonic and adult neural stem/progenitor cells. (A) UMAP plot showing the cell type composition of E18 mouse brain and (B) relative diagnostic cell marker enrichment in term of expression and percent of positive cells. (C) Feature plot of selected genes: Pax6, Eomes (Tbr2), Neurod2, Patz1. (D) Violin plot showing Patz1 enrichment in Radial Glia and Intermediate Progenitor cell types. (E) Experimental scheme used by Belenguer et al. (2021) to isolate different neural stem/progenitor cells from adult murine SVZ. (F) Heatmap and box plot showing the expression of Patz1 and selected NS/PCs markers in adult murine SVZ. RG, Radial Glia; NSC, Neural Stem Cells; IPC, Intermediate Progenitor Cells; APC, Apical Progenitor Cell; VEC, vascular endothelial cell; Ex, Excitatory; Inh, Inhibitory; qNSC, quiescent Neural Stem Cell; pNSC, primed Neural Stem Cells; aNSC, activated Neural Stem Cells; NPC, Neural Progenitor Cell; NB, Neuroblast; SVZ, Subventricular Zone.

phenol/chloroform/isoamyl alcohol (1:1), precipitated with 95\% ethanol and dissolved in TE (Tris/EDTA) buffer, pH 7.5. A set of three primers was used to detect both normal and mutant Patz1 alleles by polymerase chain reactions (PCR), as previously described (Monaco et al., 2018). The primers used were: $5^{\prime}-$ GCC TTC TTG ACG AGT TCT TC-3'/5'-CCA CAC CAT CAA AGT TGG-3' for the knockout allele; 5'-AAG CAA GTG GCT TGT GAG- $3^{\prime} / 5^{\prime}-$ CCA CAC CAT CAA AGT TGG-3' for the wild type allele.

\section{Brain Dissection}

For embryonic brain collection, C57/BL6 mice were crossed. Noon of the day on which the vaginal plug was detected was considered as 0.5 days post coitum (dpc) in the timing of the embryo collection. Timed pregnant females were sacrificed by cervical dislocation and embryos were dissected from decidual tissue in cold PBS. For each embryonic stage two different embryonic litters were analyzed. For each litter, brains from three embryos were dissected into the different territories. Corresponding brain territories of the same litter were pulled together to increase starting material. At each postnatal stage, two mice were sacrificed by cervical dislocation and brains were extracted and then dissected into the different territories. Each postnatal brain territory was analyzed separately.

The dissection was started separating the head from the body using scissors, then, a midline incision along the integument from the neck to the nose was made to expose the skull or its primordium. Any residual tissue was removed using scissors or tweezers. Before to proceed to surgery, all the dissection instruments were disinfected with $70 \%$ ethanol. Moreover, the 

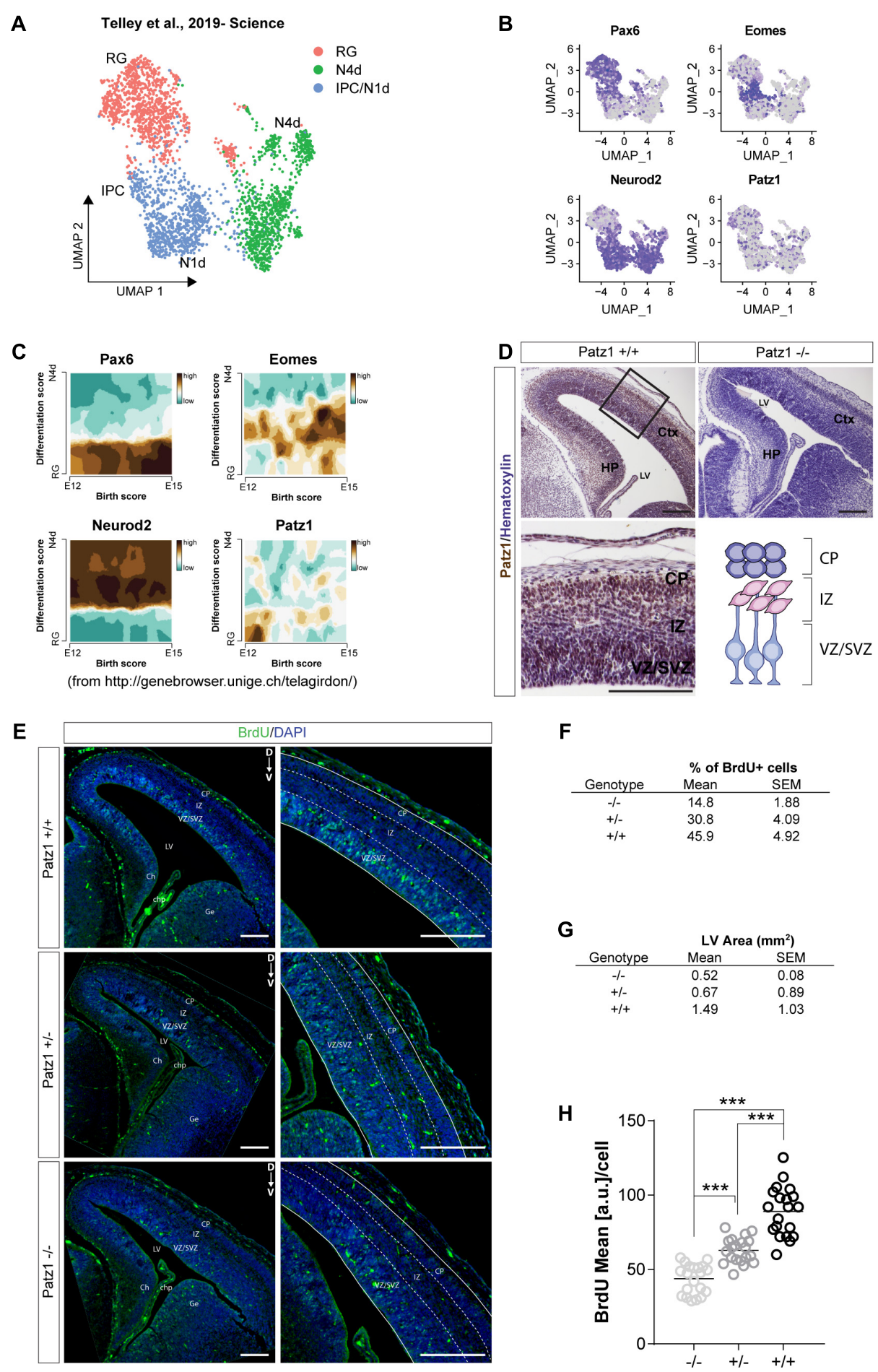

FIGURE 3 | Analysis of Patz1 expression dynamic in cortical progenitors and incorporated BrdU assay in Patz1-knockout embryonic VZ-SVZ. (A) UMAP showing cellular composition of the dataset of Telley et al. (2019), including presumptive somato-sensory cortex of E12, E13, E14, and E15 mouse embryos. (B) Feature Plots of selected progenitors and neuronal markers. (C) Trajectory plots showing the correlation between differentiation score and age (developmental stage) of selected genes. (D) Representative images and relative higher magnification of mouse embryonic brain coronal section (E15.5) immunolabeled with Patz1. (E) Representative images and relative higher magnification of BrdU immunological detection in brain coronal sections of wild type (Wt) and Patz1 mutant E15.5 embryos. Proliferating cells appear as light blue, due to overlapping of blue (DAPI) and green (BrdU) colors. (F,G) Tables showing the percentage of BrdU ${ }^{+}$cells in the VZ/SVZ (F) and lateral ventricle area (G) according to genotype. (H) Dot Plot showing the BrdU mean intensity level per cell in the different genotypes. The significant differences are indicated by asterisks. ${ }^{* * *} P<0.001$, as assessed by ANOVA followed by Tukey's multiple comparisons test. Scale bars: $100 \mu \mathrm{m}$. RG, radial glia, IPC, intermediate progenitors; N1d, 1 day old Neurons; N4d; 2 days old neurons; CP, Cortical Plate; IZ, intermediate zone; VZ/SVZ; ventricular zone/subventricular zone; LV, lateral ventricle, Ch, cortical hem; Chp; choroid plexus; Ge; ganglionic eminence. 
instruments were sterilized with $70 \%$ ethanol before the removal of each tissue in order to avoid contaminations.

\section{RNA Extraction and Reverse Transcription-Quantitative Polimerase Chain Reaction (RT-qPCR)}

Five hundred microliter of Trizol reagent (Sigma, St. Louis, MO, United States) were added to each sample, except for adult cerebellum and mesencephalon in which $1 \mathrm{ml}$ was added, for subsequent RNA extraction according to the manufacturer's protocol. Recovered RNA was reverse transcribed using M-MuLV Reverse Transcriptase (Roche Diagnostics). qPCR was performed with SYBR Green PCR Master Mix (Life Technologies) under the following conditions: $10 \mathrm{~min}$ at $95^{\circ} \mathrm{C}$, followed by 40 cycles $\left(15 \mathrm{~s}\right.$ at $95^{\circ} \mathrm{C}$ and $1 \mathrm{~min}$ at $60^{\circ} \mathrm{C}$ ). Each reaction was performed in duplicate in at least three independent experiments. The $2^{-\Delta \Delta C t}$ method (Livak and Schmittgen, 2001) to calculate the relative expression levels (fold change) was used. Amplification of two housekeeping genes, Glucose-6-Phospate Dehydrogenase (G6PD) and Ribosomal Protein S9 (RPS9) genes, was used to normalize the amount of cDNA. For analysis of brain territories in Figure 1, E11.5 or E14.5 samples have been used alternatively as calibrators to calculate the relative expression. For analysis of neurospheres, both proliferating and differentiated in Figure 6, one of control samples, randomly chosen and different for each independent experiment, was used as calibrator to calculate the $\Delta \Delta$ Ct. Sequences for forward $(\mathrm{Fw})$ and reverse $(\mathrm{Rv})$ primers were as follows: Patz1 $\mathrm{Fw}=5^{\prime}-\mathrm{GAG}$ CTT CCC CGA GCT CAT-3'; Patz1 Rv = 5'-CAG ATC TCG ATG ACC GAC CT$3^{\prime} ;$ Nanog Fw $=5^{\prime}-$ GCC TCC AGC AGA TGC AAG-3'; Nanog $\mathrm{Rv}=5^{\prime}$-GGT TTT GAA ACC AGG TCT TAA CC-3'; Nestin $\mathrm{Fw}=5^{\prime}-\mathrm{GAG}$ AAG ACA GTG AGG CAG ATG AGT T3'; Nestin Rv = 5'-GCC TCT GTT CTC CAG CTT GCT-3'; Tubb3 Fw $=5^{\prime}-$ GAA TGA CCT GGT GTC CGA GT-3'; Tubb3 $\mathrm{Rv}=5^{\prime}-\mathrm{CCG}$ ATT CCT CGT CAT CAT CT $-3^{\prime} ;$ G6pd Fw $=5^{\prime}-$ CAG CGG CAA CTA AAC TCA GA-3'; G6pd Rv $=5^{\prime}-\mathrm{TTC}$ CCT CAG GAT CCC ACA C-3'; Rps9 Fw $=5^{\prime}-\mathrm{CTG}$ GAC GAG GGC AAG ATG AAG $c-3^{\prime}$; Rps9 Rv $=5^{\prime}$ - TGA CGT TGG CGG ATG AGC ACA-3'.

\section{Bromodeoxyuridine Pulse-Labeling and Detection}

Heterozygous Patz1-mutant mice were mated and vaginal plug monitored each day to establish the coitum day. At 15.5 days dpc pregnant mice received a single intra-peritoneal injection of $5^{\prime}$-bromo-2'-deoxyuridine (BrdU) $(50 \mathrm{mg} / \mathrm{kg})$ and, $2 \mathrm{~h}$ later, they were euthanized. One embryo for each genotype was extracted, post-fixed in $4 \%$ paraformaldehyde for $24 \mathrm{~h}$ and then kept in $70 \%$ ethanol at $4^{\circ} \mathrm{C}$ before to be processed for immunofluorescence analysis. Embryos were then dehydrated through ascending ethanol, washed in Toluene and TolueneParaffin 1:1, included in paraffin and then sectioned using a microtome to undergo histological analyses (Giorgio et al., 2014). For details see elsewhere (Q4Lab, 2016). Briefly, cutting angle and thickness have been set, respectively, at 5 and
$8 \mu \mathrm{m}$. Paraffin sections have been dewaxed in xylene (prewarmed at $55-65^{\circ} \mathrm{C}$ ) twice for $5 \mathrm{~min}$ and then rehydrated through sequential steps in gradually less concentrated ethanol solutions and rinsed in tap water, at the end. Slides have been heated in a $10 \mathrm{mM}$ sodium citrate $\mathrm{pH} 6.0$ in a microwave to expose the antigens (Pisapia et al., 2020). Tissue sections have been incubated $1 \mathrm{~h}$ at R.T. with a solution $1 \%$ milk, 10\% FBS, 1\% BSA, 1X Sodium Azide, 0,5\% Tween in PBS 1X and then O.N. at $4^{\circ} \mathrm{C}$ with a rat-monoclonal BrdU antibody (Novus Biologicals NB500-169) at dilution 1:400. Sections have been rinsed and incubated $1,5 \mathrm{~h}$ at R.T. with a Donkey $\alpha$-rat Alexa Fluor- 488 secondary antibody (Molecular Probes A21208) at 1:400 dilution, counterstained with Hoechst 1:5,000 and then mounted.

\section{Patz1 Immunological Detection}

Sections were dewaxed in xylene, hydrated in graded series of alcohol and subjected to heat-induced antigen retrieval (Guadagno et al., 2017). After blocking endogenous peroxidase activity, the tissue was incubated for $1 \mathrm{~h}$ at R.T. with the blocking solution previous described and then with our previously characterized polyclonal antibody able to recognize both human and mouse Patzl proteins (Valentino et al., 2013a) at a dilution 1:40 (V/V) O.N. Subsequently, the slices were rinsed and incubated with the biotinylated secondary antibody, at room temperature, for $30 \mathrm{~min}$. The bound antibody complexes were stained for 3-5 min with diaminobenzidine, and slides were then counterstained with hematoxylin (30 s), dehydrated and mounted.

\section{Neurosphere Culture, Proliferation, and Differentiation Assays}

Before surgery all the dissection instruments have been disinfected with $70 \%$ ethanol. Adult mice or pregnant females were euthanized. SVZ dissection from 4 wild type, $3 \mathrm{Patz1}^{+/-}$ and $2 \mathrm{Patz}^{-/}$- adult mice or total forebrain dissection from 6 wild type, $6 \mathrm{Patz}^{+/-}$and $2 \mathrm{Patz1}^{-/-} 13.5 \mathrm{dpc}$ embryos was carried out under stereoscopic control in ice-cold disodium phosphate buffer solution supplemented with glucose $0.6 \%$ (PBSg). The dissected samples were transferred into a new sterile cold PBSg buffer and brought under the hood where they were mechanically dissociated in single cell suspension.

For each sample, cells were counted and plated at the density of 3,000 cells $/ \mathrm{cm}^{2}$ in $14-16 \mathrm{ml}$ of serum-free medium comprising 1:1 mixture of DMEM and F12 supplemented with $2 \mathrm{mM}$ glutamax, N2 supplement (1:100), B27 minus Vitamin A (1:50) (Invitrogen), $20 \mathrm{ng} / \mathrm{ml}$ EGF (Sigma), 20 $\mathrm{ng} / \mathrm{ml} \mathrm{bFGF} \mathrm{(Sigma)} \mathrm{and} \mathrm{penicillin/streptomycin} \mathrm{(1:100),} \mathrm{into}$ T75 neurosphere-flasks in a prewarmed culture medium, to obtain a single embryo cell culture. $1 \times 10^{5}$ and all cells recovered from dissection were plated for embryonic and adult neurospheres, respectively. The number of neurospheres/brain were counted under microscope after 4 days in culture for embryonic cells and 7 days for adult ones, and their diameter measured by ImageJ. Only neurospheres $>40 \mu \mathrm{m}$ in diameter were considered (Scopa et al., 2020). Primary neurospheres 
(P0) were then disaggregated by incubating with TripleExpress dissociation reagent (Invitrogen) for $5 \mathrm{~min}$ at room temperature. After the incubation, the dissociation reagent was aspirated, the neurosphere were mechanically dissociated and replated $\left(1 \times 10^{5} /\right.$ plate $)$ to form secondary neurospheres $(\mathrm{P} 1)$. Initial selfrenewal activity was evaluated by counting the number of $\mathrm{P} 1$ neurospheres/cell seeded ${ }^{*} 100$.

For differentiation assay, neurospheres at passage 3 were dissociated to obtain single cell suspension, as described above and plated on poly-D-lysine coated multiwell plates at a density of 50,000 cells $/ \mathrm{cm}^{2}$. Cells were grown in absence of growth factors for 6 days in a 1:1 mixture of Neurobasal and DMEM/F12 medium supplemented with B27 plus Vitamin A (1:50), $2 \mathrm{mM}$ glutamax (Invitrogen) and penicillin/streptomycin (1:100). Half of the differentiation medium was replaced every other day.

\section{Image Quantification}

Image/Fiji software was used to perform image analysis (Rueden et al., 2017). To compute the percent of $\mathrm{BrdU}^{+}$cells (Figure 3F), 10 different images were analyzed per sample by defining the number of $\mathrm{BrdU}^{+}$cells over Hoecsht ${ }^{+}$cells on 5 different regions of interest (ROIs) manually set on VZ/SVZ using Cell Counter ImageJ Plug-in. The lateral ventricle area was computed over 5 different images per sample spanning from anterior to posterior regions, by segmenting Hoecsht signal and automatically defining ROIs over segmentation using ImageJ default threshold algorithm (Figure 3G). BrdU intensity signal per cell was computed by segmenting single positive nuclei of 3 different images per sample using ImageJ MaximalEntropy threshold function and computing the Mean Intensity arbitrary unit (a.u.) score using the Analyze Particle function. Representative quantifications of this analysis are shown in the dot plot in Figure $3 \mathrm{H}$.

To compute the percent of $\beta$-III-tubulin ${ }^{+}$cells (Figure 6F), 5 different images were analyzed per sample by defining the number of $\beta$-III-tubulin ${ }^{+}$cells over Hoecsht ${ }^{+}$cells using Cell Counter ImageJ Plug-in. NeuroJ Plung-in was used to perform neurite guided tracing and perform number (Figure 6H), length (Figure 6I), and fluorescence score (Figure 6J) analysis of 3-5 neurons per image for at least 3 images per sample.

\section{Statistical Analysis}

Pearson correlation test was performed among the Patz1 expression levels detected and the age of the analyzed samples (Figure 1) and between Patz1 and Nanog expression in Patz1 $1^{+/+}$ and $\mathrm{Patz}^{+/-}$adult neurospheres (Supplementary Figure 1). Ordinary one-way analysis of variance (ANOVA) followed by Tukey's multiple comparisons test was applied for comparison of all the other sets of data. The values analyzed are the average \pm SD of at least two biological replicates and three independent experiments. All tests were assessed using GraphPad Prism 7 software, La Jolla (CA), United States. Statistical significance was indicated by ${ }^{*} p<0.05 ;{ }^{* *} p<0.01 ;{ }^{* * *} p<0.001$; $^{* * * *} p<0.0001$ vs. wild type control when not differently specified in the figures by linking lines.

\section{RESULTS}

\section{Patz1 Is Expressed During Mouse Brain Development and Turns Down in Adult Brain}

To get insight into Patzl expression pattern during embryonic development, we took advantage of the Expression Atlas publicly available dataset containing RNA-seq data from different tissues across multiple timepoints during both embryonic and postnatal development (Figures 1A,B). As shown in Figure 1A, Patz1 expression is frequently higher during embryogenesis than in postnatal and adult stages, with the strongest expression of Patz1 in embryonic brain respect to the other tissues analyzed (heart, liver, ovary, and testis). Interestingly, during brain development, a decreasing trend of Patz1 expression with respect to time, from E10.5 to P63, is quite evident (Figure 1B).

To confirm and expand these findings, we analyzed, by means of qPCR, Patzl transcript distribution levels in specific mouse brain territories at different time points during both embryonic $(11.5,14.5$, and $17.5 \mathrm{dpc})$ and postnatal (14 days and adults) life. The results are shown in Figure 1C and confirm the existence of a temporal Patz1 expression gradient, with higher levels of expression at early embryonic stages, that decrease during embryogenesis and even more during postnatal (P14) and adult life, when Patz1 expression reaches the minimum measured level. This trend is detectable in the whole brain (Pearson correlation index $=-0.6249 ; p=0.0014)$ and also in the specific structures analyzed, as reported in Figures 1C-E. Conversely, there is no preferential spatial distribution at the different embryo and adult stages. Interestingly, looking at the relative expression of Patz1 in the adult, a higher expression level was detected in telencephalic structures (olfactory bulbs, cortex, and hippocampus), in which neurogenesis mainly occurs after birth, respect to the most posterior ones (Figure 1C).

\section{Patz1 Is Enriched in Proliferating Neural Progenitor Cells Inside the SVZ and Appears Involved in Their Maintenance}

Given the complex cellular heterogeneity and temporal dynamics of embryonic and adult brain tissue, we took advantage of publicly available single-cell and bulk RNA sequencing datasets to get insight into Patz1 expression level in neural progenitors during brain development. By analyzing the $10 \times$ Genomics 10K E18 Brain cells dataset, we found an enrichment of Patz1 expression in early apical radial glia/neural stem cells (RG/NSCs) and intermediate progenitors (IPCs) (Figures 2A-D). We also examined Patzl expression in a dataset of bulk RNA sequencing from sorted types of progenitors from mouse adult SVZ (Belenguer et al., 2021), finding that Patz1 was expressed in the entire proliferating lineage with an enrichment in neural progenitor cells (NPCs) and neuroblasts (NBs) (Figures 2E,F). A further analysis, on single-cell RNA sequencing of sorted progenitor cells belonging to presumptive somato-sensory cortex of E12, E13, E14, and E15 mouse embryos (Telley et al., 2019), confirmed an enrichment of Patz1 expression 
during development in early apical radial glia/neural stem cells (RG/NSCs), mainly at the E12 stage (Figures 3A-C). Accordingly, Patz1 immunodetection at E15.5 showed a strong immunoreactivity in the cortical plate as well as in the VZ/SVZ of wild type embryo (Figure 3D). As expected, no Patz1 immunosignal was detected in corresponding brain sections of null mutants (Figure 3D). All these data point out to a possible function of Patz1 during embryonic neurogenesis. Then, to have a first indication of the impact of Patzl depletion on proliferating cells in the periventricular area, we examined the in vivo proliferation inside the SVZ of $15.5 \mathrm{dpc}$ Patz1-null and heterozygous mouse embryos, compared to wild type control, using a saturation BrdU (5'-bromo-2'-deoxyuridine) pulselabeling method (Kuhn et al., 1996; Hayes and Nowakowski, 2002) that could label the entire pool of proliferating NS/PCs within a $12 \mathrm{~h}$-period (Figure 3E). BrdU is a thymidine analog that incorporates into dividing cells during DNA synthesis. BrdUlabeling appears weaker and unevenly distributed in the SVZ of Patz1-null and heterozygous mutants compared to control brains. Quantitative analysis indicates a reduction of the number of proliferating cells (Figure 3F) and a significant decrease of BrdU incorporation level (Figure $3 \mathbf{H}$ ) coupled to an overall reduction of lateral ventricle area (Figure 3G) in both Patz1 mutants compared with control. Interestingly, the heterozygous shows mean values lower than wild type and higher than Patz1 null mutant for all parameters analyzed.

\section{Reduced Proliferative Capacity in Patz1-Knockout-Derived Neurospheres}

To effectively measure the number of NS/PCs and their proliferation rate, we performed neurosphere assays on both Patz1 mutant and wild type brains. In this assay NS/PCs derived from SVZ of $\mathrm{Patz1}^{-/-}, \mathrm{Patz1}^{+/-}$and wild type adult mice (more than 1 month up to 21 months of age) as well as from $13.5 \mathrm{dpc}$ embryonic telencephalon (from which SVZ arises), were allowed to proliferate in culture at very low density to form spheroid cell aggregates called neurospheres. The number of neurospheres was determined after 4 days in culture for embryonic and 7 days for adult neurospheres (Figures 4A, 5A). Under these conditions, primary neurosphere colonies are derived from single cells and can be used as a good model of the number of in vivo NS/PCs (Morshead et al., 2003; Coles-Takabe et al., 2008). In agreement with in vivo BrdU labeling data (Figures $3 \mathrm{E}-\mathbf{H}$ ), the number of primary neurospheres (P0) isolated from $\mathrm{Patz1}^{-/-}$ embryonic telencephalons was significantly lower than wild type counterparts (mean difference -1208 ; 95\% CI of diff. -2377 to -39.62) (Figures 4B,C). The diameter of each neurosphere, an indirect measure of the ability of cells to grow and proliferate, was also determined, showing a significant reduction in neurospheres derived from Patz1-1- embryos compared to the wild type (mean difference $-12,36$; $95 \%$ CI of diff. -24.48 to -0.2338 ) (Figure 4D). Furthermore, to assess the initial NS/PC selfrenewal capability, primary neurospheres were dissociated into single cells after 4-7 days in culture and 10,000 cells per sample were cultured as above to form secondary neurospheres (P1) that were counted (day 8 and 14 for embryonic and adult neurospheres, respectively). As shown in Figure 4E, we observed a significant reduction (mean difference $=-1.787$; 95\% CI of diff. -3.261 to -0.3126 ) in the number of P1 neurospheres from $\mathrm{Patz}^{-/-}$embryos compared to wild type controls, indicating the impairment of the self-renewal ability of Patz1-null NS/PCs. The reduction of the number (mean difference $-332.9 ; 95 \%$ CI of diff-561.5 to -104.4 ), the diameter (mean difference $-81.52 ; 95 \%$ CI of diff. -145.8 to -17.26 ) and the self-renewal capability (mean difference -3.425 ; 95\% CI of diff. -6.053 to -0.7973) of NS/PCs lacking Patz1 was also evident in the SVZ of adult brain (Figure 5). Even though no significant difference was observed in the number and size of neurospheres from the heterozygous $\mathrm{Patz1}^{+/-}$brains, compared to both Patz1 $1^{-/-}$ and wild type samples, data from both embryonic and adult samples suggest for $\mathrm{Patz1}^{+/-}$heterozygotes an intermediate trend between Patz1-null mutants and wild type controls (Figures 4C,D, 5C,D). Noteworthy, both embryonic and adult Pat $1^{+/-}$NS/PCs showed a self-renewal capability significantly higher than Patz1-null homozygotes and comparable to wild type (Figures $4 \mathrm{E}, 5 \mathrm{E}$ ).

Overall, these data provide evidence for a role of Patz1 in the maintenance, proliferation and self-renewal capability of NS/PCs at both embryonic and adult stages.

\section{Downregulation of Stem Cell Related Genes and Enhanced Neuronal Differentiation in Patz1-Knockout-Derived Neurospheres}

RNA extracted from embryo-derived neurospheres, after at least three passages in culture (Figure 4A), under proliferating conditions, was used to analyze expression of genes characteristic of stemness (Nanog) and neural progenitor (Nestin) phenotype in proliferating neurospheres, or neuronal (Tubb3) cells. Nanog is a DNA binding homeobox transcription factor involved in embryonic stem cell proliferation, renewal, and pluripotency, which is a general marker of stemness; Nestin, neuroepithelial stem cell protein, is an intermediate filament protein that has generally been considered to be a marker of NS/PCs and is crucial for their self-renewal and survival; while Tubb3, coding for $\beta$-IIItubulin, correlates with neuronal differentiation (Zhang and Jiao, 2015). In agreement with the above data, both Nanog and Nestin genes were significantly downregulated in homozygous Patz1null NS/PCs with respect to wild type (Figures 6A,B), confirming also at molecular level that Patz1 inactivation strongly affects the stem/progenitor phenotype. Moreover, Patz1-null heterozygous neurospheres also showed a significant downregulation of Nanog and Nestin expression with respect to the control ones. However, Nestin expression levels of $\mathrm{Patz}^{+/-}$NS/PCs settle in the middle between $\mathrm{Patz1}^{-/-}$and wild type NS/PCs, whereas Nanog expression of $\mathrm{Patz1}^{+/-}$NS/PCs was as low as in the homozygous Patz1-null neurospheres. These results might suggest a significant reduction of the Nestin ${ }^{+}$and $\mathrm{Nanog}^{+}$cells in Patz1-knockout NS/PCs compared to wild type controls. However, according to the role of Patz1 as a positive regulator of Nanog expression in mouse embryonic stem cells (Ow et al., 2014), Patzl might activate Nanog expression also in NSCs 

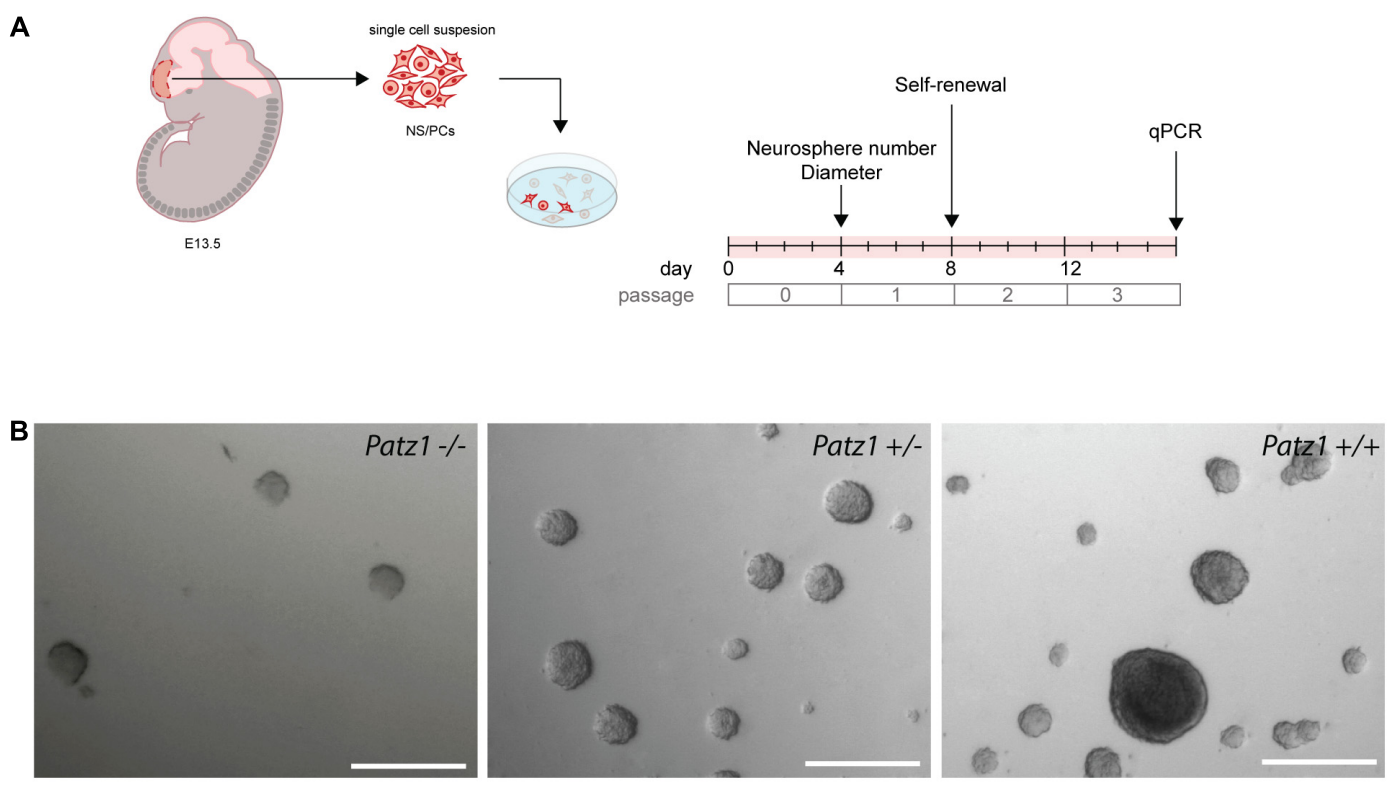

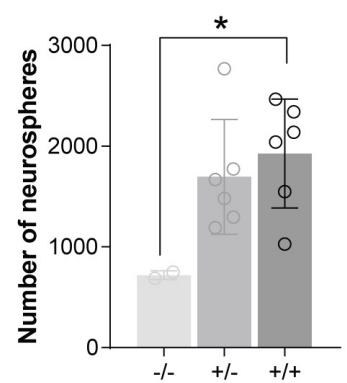

D

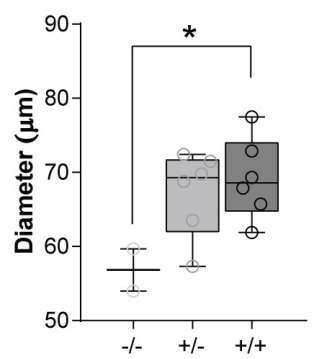

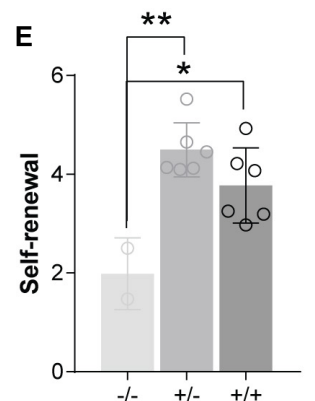

FIGURE 4 | Ex vivo proliferation assay of 13.5 dpc embryonic telencephalon-derived neurospheres. (A) Experimental scheme showing the brain region microdissected to produce neurospheres, and relative experiment timeline. (B) Representative images of neurospheres from Patz1-/-, Patz1+/- and wild type embryos. Scale bars $=100 \mu \mathrm{m}$. (C) Bar/dot plot (means \pm SD) showing the number of primary neurospheres (P0) obtained by plating $1 \times 10^{5}$ cells/embryo obtained from the dissected tissue. (D) Box dot/plot showing the diameter of P0 neurospheres (median \pm min to max). (E) Neurosphere self-renewal capacity (number of neurospheres of the first-generation (P1)/plated cells ${ }^{*} 100$ ). All the significant differences are indicated by asterisks. ${ }^{\star} P<0.05 ;{ }^{\star \star} P<0.01$, as assessed by ANOVA followed by Tukey's multiple comparisons test.

and the reduced levels of Nanog could be partly due to the direct modulation of Nanog promoter by Patz1. Consistently, Patz1 and Nanog gene expression were highly correlated in adult neurospheres as well (Pearson correlation index $=0.9$; $p=0.0069$ ) (Supplementary Figure 1).

Conversely, the expression of Tubb3 did not change significantly in the three groups (Figure 6C), even though a slight increasing trend was visible among the different genotypes from $\mathrm{Patz}^{+/+}$to $\mathrm{Patz}^{-/-}$proliferating neurospheres, which might suggest an accelerated differentiation into neurons of the Patz1-null neurospheres. To verify this hypothesis, neurospheres were cultured for 6 days in differentiating conditions and neuronal differentiation was confirmed by $\beta$-IIItubulin immunodetection (Figure 6D). Tubb3 transcript levels after neurospheres differentiation did not show any significant difference among the different genotypes (Figure 6E), as well as the number of $\beta$-III-tubulin ${ }^{+}$cells detected (Figure 6F) and the mean fluorescence level per neuron (Figure 6G). However, both number and length of neurites were higher in $P a t z 1^{-/-}$than in both Patz1 $1^{+/-}$and Pat $z 1^{+/+}$cells (Figures $6 \mathbf{H}-\mathbf{J}$ ), indicating that Patz1-null neurospheres can go toward an enhanced neuronal differentiation compared to control. Consistent with the genotype, Patz1 expression in heterozygous was about half the level of wild type cells, while it was not detected at all in Patz1null samples (Figure 6K). Interestingly, its expression was lower in differentiated than in proliferating cells, once again supporting a role of Patz1 in counteracting neuronal differentiation.

\section{DISCUSSION}

Growing evidences suggest that Patzl and stemness are closely associated. Patz1 is required for pluripotency maintenance of mouse embryonic stem cells (ESCs), by 


\section{A}

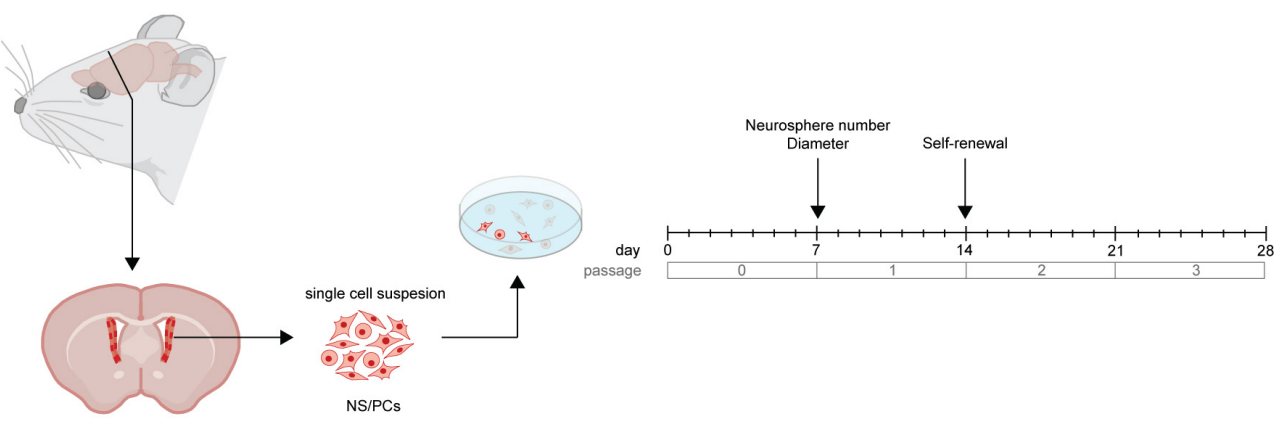

B
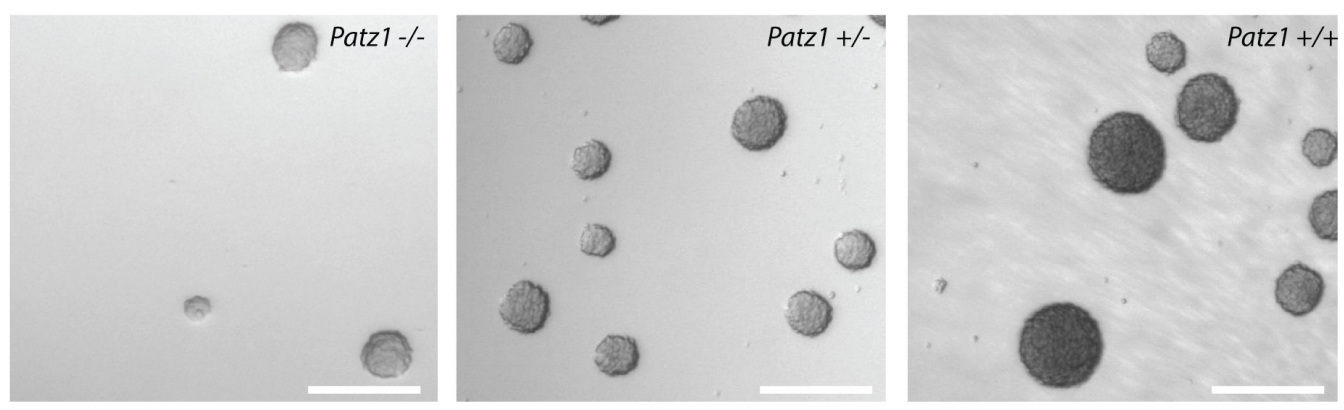

C

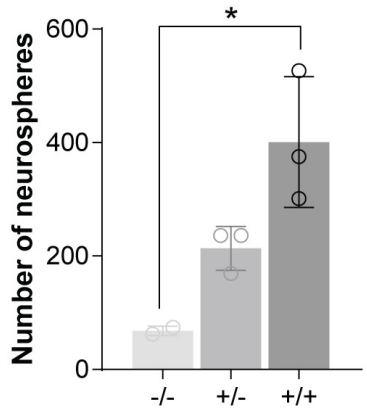

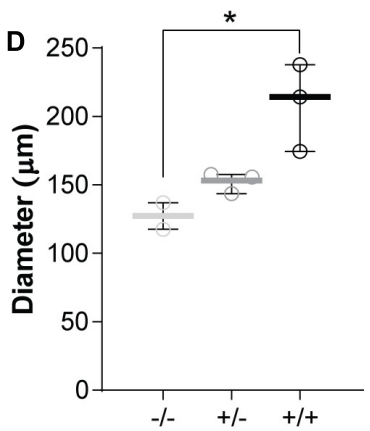

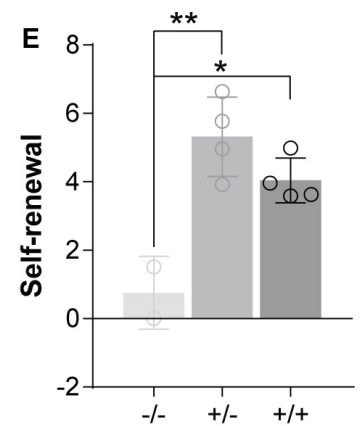

FIGURE 5 | Ex vivo proliferation assay of SVZ-derived NS/PCs from adult mice. (A) Experimental scheme showing the brain region microdissected to produce neurospheres and relative experiment timeline. (B) Representative images of neurospheres from Patz $1^{-1-}$, Patz $1^{+/-}$and wild type mice. Scale bars $=100 \mu \mathrm{m}$. (C) Bar/dot plot (means \pm SD) showing the number of primary neurospheres (PO) obtained from the adult brains by plating all the cells recovered by SVZ dissection. (D) Box dot/plot showing the diameter of the PO neurospheres (median \pm min to max). (E) Neurosphere self-renewal capacity (number of neurospheres of the first-generation (P1)/plated cells * 100). All the significant differences are indicated by asterisks. ${ }^{\star} P<0.05 ;{ }^{* *} P<0.01$, as assessed by ANOVA followed by Tukey's multiple comparisons test.

regulating expression of pluripotency master genes Pou5f1 and Nanog (Ow et al., 2014), and its gene dosage is crucial for an efficient reprogramming of mouse induced pluripotent stem (iPS) cells (Ma et al., 2014). Moreover, during mouse embryonic development, Patz1 gene expression has been detected in actively proliferating neuroblasts of the periventricular and subventricular neocortical neuroepithelium, and $\mathrm{Patz1}^{-/-}$embryos showed anatomical reduction of the SVZ (Valentino et al., 2013b).

Here, we confirmed and expanded these previous results, revealing a temporal decreasing expression gradient of Patz1 gene during mouse brain development. Indeed, Patz1 transcription levels in the brain decrease during embryogenesis and even more at postnatal stages, reaching the minimum levels in adult mice. In adults, higher Patz1 expression levels correspond to brain structures characterized by still active neurogenesis, such as olfactory bulbs, cortex and hippocampus. The maintenance of high Patz1 expression in the adult hippocampus, a region that is essential for many forms of learning, memory and mood regulation (Christian et al., 2014), suggests that aberrant expression of PATZ1 could contribute to neurological and psychiatric disorders. Indeed, adult-generated neurons and the dynamic regulation of neurogenesis by epigenetic factors are relevant for neuropsychiatric disorders, including depression, 
A

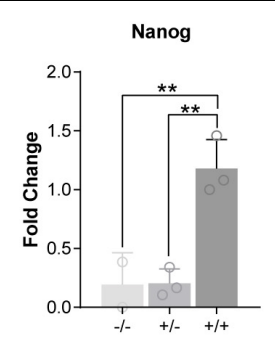

D

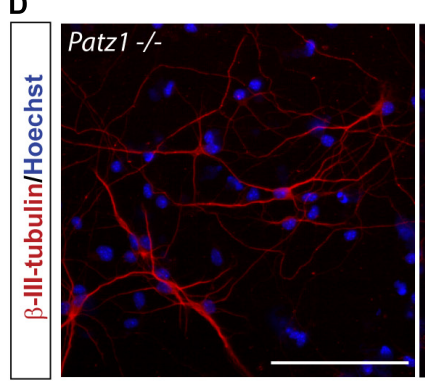

E

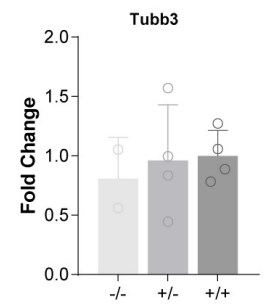

H

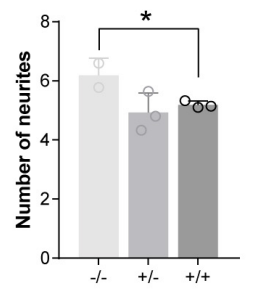

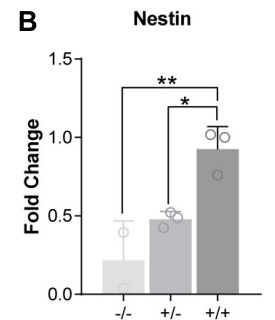

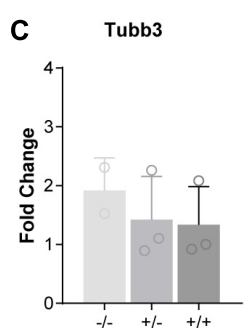

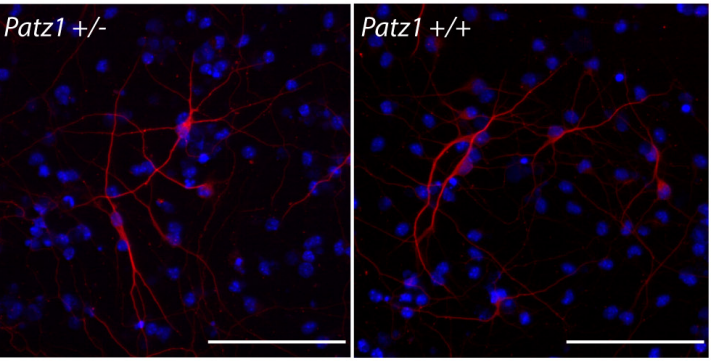

$\mathbf{F}$
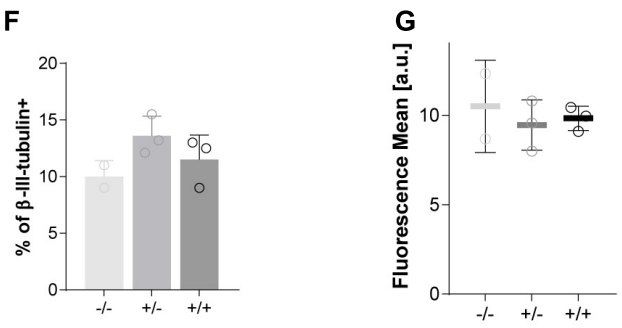

I
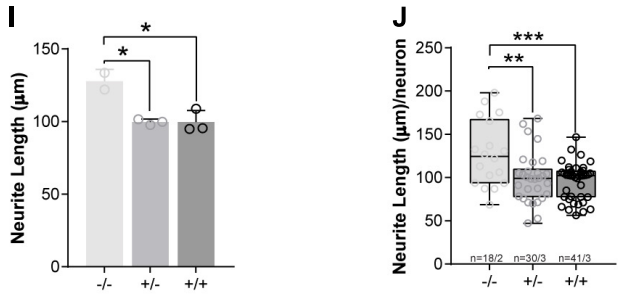

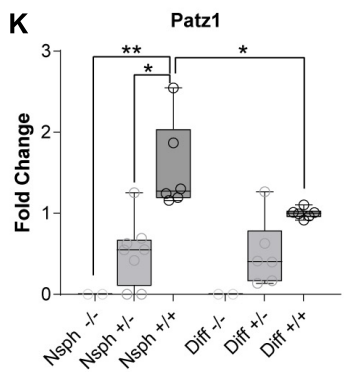

FIGURE 6 | Real time analysis of gene expression and neuronal differentiation in proliferating neurospheres and differentiated cells. (A-C) Relative expression of the genes indicated at the top of each graph is expressed as fold changes with respect to the level of a wild type control, randomly chosen and different for each independent experiment. Mean values \pm SD of at least three independent experiments performed in duplicate on samples derived by 2 Patz $1^{-/-}, 3$ Patz $1^{+/-}$and 3 wild type embryo-derived neurospheres are reported. (D) Representative images of differentiated cells immunolabelled with $\beta$-III-tubulin neuronal marker. (E) Relative expression of Tubb3 in differentiated cells as assessed by RT-qPCR. (F-J) Quantitative analysis of the experiment shown in D plotted to show the percentage of $\beta$-III-tubulin ${ }^{+}$cells (neurons) $\mathbf{( F )}$, the mean fluorescence of $\beta$-III-tubulin intensity $(\mathbf{G})$, the number $(\mathbf{H})$ and length $\mathbf{( I )}$ of neurite branches, and the neurite length/neuron ratio (J). (K) Relative expression level of Patz1 in proliferating neurospheres (Nsph) and after 6 days in differentiation medium (Diff). ${ }^{*} p<0.05 ;{ }^{* *} p<0.01$; ${ }^{* \star *} p<0.001$ vs. wild type control, if not diversely indicated by connecting line, as assessed by ANOVA followed by Tukey's multiple comparisons test. 
addiction, and epilepsy (Hsieh and Eisch, 2010). Consistently, PATZ1 has been identified as one of the differentially regulated key genes in a form of depression (Xu et al., 2015). Moreover, very recently, PATZ1 has also been associated to Parkinson's disease, consistent with its possible contribution to neurodegenerative diseases (Schilder and Raj, 2020). However, no studies have so far specifically investigated the role of PATZ1 in NS/PCs.

We now showed an in vivo analysis of BrdU incorporation in embryonic brain of Patz1-null mutant mice, confirming previous observations (Valentino et al., 2013b) and suggesting a different distribution and lowered number of proliferating cells inside the SVZ of Patz1-null mutants compared to wild type embryos. Quantitative and qualitative analysis of neurospheres in culture is extremely helpful in studying both self-renewal and differentiation potential of NS/PCs and dissecting mouse mutant neural phenotype (Morshead et al., 2003; Coles-Takabe et al., 2008; Liguori et al., 2009). By using this approach on our Patz1-knockout mouse model, we explored the role of Patz1 in self-renewal and proliferation of NS/PCs. Albeit with the limitation of a small number of Patz1 $1^{-/-}$samples, due to the high and early embryonic lethality of this genotype in our current mouse colony, the data indicate that Patzl contributes to proliferation and self-renewal of NS/PCs inside the SVZ, likely playing a role in the early development of the mouse brain, and lately in the maintenance of the adult neurogenic niches. Indeed, evidence for a role of Patz1 in the maintenance and proliferation of NS/PCs was corroborated by impairment of the self-renewal of Patz1-null NS/PCs in both embryonic and adult ex vivo neurosphere cultures derived from telencephalon and SVZ, respectively. Consistent with neurosphere functional data, the gene expression analysis in proliferating neurospheres also suggests that Patz1 inactivation might strongly impair the ability of these NS/PCs to maintain a stemness phenotype. Indeed, the Patz1-null neurospheres express significantly lower levels of stemness and neural progenitor markers, such as Nanog and Nestin, respectively, while the neuronal marker Tubb3 does not seem to be affected, indicating a specific role of Patz1 in their proliferation and self-renewal. These data are consistent with recent results in other cell types, such as rat thyroid cancer stemlike cells, in which Patz1 expression enhances self-renewal ability (Vitiello et al., 2019).

We also provided a first evidence of a negative role for Patz1 in the differentiation of embryonic neurospheres, showing enhanced neuronal differentiation in $\mathrm{Patz}^{-/-}$compared to $\mathrm{Patz1}^{+/-}$and $\mathrm{Patz1}^{+/+}$cells. Moreover, differentiated wild type cells had significantly lower levels of Patz1 than non-differentiated neurospheres, supporting its opposing role in neurosphere differentiation. Additional neurosphere differentiation experiments are underway to investigate possible additional roles of Patz1 in neuro-glial differentiation. Consistently, a recent study linked PATZ1 to neuron and glia differentiation through its interaction with the variant polycomb repressor complex 1 component PCGF1, which also interacts with NANOG and OCT4, and contribute to the maintenance of the undifferentiated phenotype of NT2 teratocarcinoma cell line that, upon treatment with all-trans retinoic acid, can differentiate to both neuron and glia (Oliviero et al., 2015).
Moreover, it has recently been shown that during neural differentiation, Nanog prevents the upregulation of genes important for neural specification (Barral et al., 2019). As a further support, our previous studies have associated PATZ1 to cell plasticity, being it involved in both epithelial-mesenchymal and proneural-mesenchymal transition (Chiappetta et al., 2015; Fedele et al., 2019).

It is well known that epigenetic mechanisms, including DNA and histone modifications, play critical roles in different stages of neurogenesis, and that aberrant epigenetic regulation also contributes to the pathogenesis of various brain disorders (Yao et al., 2016). PATZ1 belongs to a large family of transcription factors, named POZ-ZF or POK, including BCL6, PLZF, TAZ1, and others, in which the POZ/BTB domain mediates protein-protein interactions, allowing the recruitment of histone deacetylases through co-repressor complexes (Costoya, 2007). PATZ1 is indeed an epigenetic regulator acting through the recruitment of repressor complexes that modulate histone deacetylase levels to the promoter of different genes (Sakaguchi et al., 2010; Cho et al., 2012). Based on these findings, PATZ1 may be one of the key epigenetic factors that, by remodeling chromatin through the recruitment of histone deacetylases, is implicated in neurogenesis and, consequently, neurodegenerative and neuropsychiatric disorders. In this frame, the relatively high expression of PATZ1 in the hippocampus is particularly significant, where adult NSCs generate granular cells that confer the plasticity necessary for memory and behavior (Jessberger et al., 2009; Sahay et al., 2011). PATZ1 in these cells could be crucial for such plasticity, accounting for its involvement in depression ( $\mathrm{Xu}$ et al., 2015). Indeed, hippocampus is an important anatomical area associated with depression and studies have shown that some antidepressant can treat depression by changing the plasticity of the hippocampus (Xu et al., 2020). Future experiments are needed to verify such hypothesis.

A tight link exists between NSCs and glioblastoma stem cells (GSCs). Glioblastoma (GBM) is the most frequent form of brain tumor in adults and is associated with a poor prognosis in both adults and children due to the poor tumor response to the limited available therapeutic options (Sturm et al., 2014). Various studies have suggested that NSCs might be the cells of origin of GBM, which arises from migration of mutated astrocyte-like NSCs (Alcantara Llaguno et al., 2009; Lee et al., 2018; Altmann et al., 2019). We have previously shown that PATZ1 is overexpressed in both adult and pediatric GBM compared to normal glial cells (Guadagno et al., 2017; Passariello et al., 2019), and that it is specifically expressed in GSCs, where it is associated with the proneural phenotype (Guadagno et al., 2017). Based on the results emerged from the present study, we could hypothesize that PATZ1 overexpression may contribute in maintaining the self-renewal and proliferative capacity of GSCs, likely favoring symmetric over asymmetric stem cell division. It is in fact known that both HMGA1 and p53, two PATZ1 interactors (Fedele et al., 2012; Valentino et al., 2013a; Keskin et al., 2015), are deeply involved in the balance between symmetric and asymmetric stem cell division, which plays a key role in the expansion of the stem cell pool in both NSCs and GSCs (Cicalese et al., 2009; Puca et al., 2019). Indeed, an undue increase in NSC symmetrical divisions, 
due to the disruption of molecular regulators, is the prelude to the conversion to GSCs and tumors in invertebrates (Caussinus and Gonzale, 2005). Future molecular experiments are needed to clarify this possibility.

\section{DATA AVAILABILITY STATEMENT}

The datasets presented in this study can be found in online repositories. The names of the repository/repositories and accession number(s) can be found in the article/Supplementary Material.

\section{ETHICS STATEMENT}

The animal study was reviewed and approved by the Italian Ministry of Health (prot. no. 576/10 approved on 3 February, 2011). Written informed consent was obtained from the owners for the participation of their animals in this study.

\section{AUTHOR CONTRIBUTIONS}

MF: conceptualization, project administration, and original draft preparation. GLL and SM: methodology. SM, MV, and MD: validation. GLL and MF: formal analysis and supervision. SM, MV, MD, FM, GP, and AL: investigation. CA, GLL, and MF: resources. SM, GLL, and MF: data curation. SM, GLL, LC, and MF: writing, review and editing. LC and GLL: funding

\section{REFERENCES}

Alcantara Llaguno, S., Chen, J., Kwon, C. H., Jackson, E. L., Li, Y., Burns, D. K., et al. (2009). Malignant astrocytomas originate from neural stem/progenitor cells in a somatic tumor suppressor mouse model. Cancer Cell 15, 45-56. doi: 10.1016/j.ccr.2008.12.006

Altmann, C., Keller, S., and Schmidt, M. H. H. (2019). The role of SVZ stem cells in glioblastoma. Cancers 11:448. doi: 10.3390/cancers 11040448

Alvarez-Buylla, A., and Garcia-Verdugo, J. M. (2002). Neurogenesis in adult subventricular zone. J. Neurosci. 22, 629-634. doi: 10.1523/JNEUROSCI.22-0300629.2002

Andersen, L., Gülich, A. F., Alteneder, M., Preglej, T., Orola, M. J., Dhele, N., et al. (2019). The Transcription factor MAZR/PATZ1 regulates the development of FOXP3+regulatory T cells. Cell Rep. 29, 4447-4459.e6. doi: 10.1016/j.celrep. 2019.11.089

Barral, A., Rollan, I., Sanchez-Iranzo, H., Jawaid, W., Badia-Careaga, C., Menchero, S., et al. (2019). Nanog regulates Pou3fl expression at the exit from pluripotency during gastrulation. Biol. Open 8:bio046367. doi: 10.1242/bio.046367

Belenguer, G., Duart-Abadia, P., Jordán-Pla, A., Domingo-Muelas, A., BlascoChamarro, L., Ferrón, S. R., et al. (2021). Adult neural stem cells are alerted by systemic inflammation through TNF- $\alpha$ receptor signaling. Cell Stem Cell 28, 285-299.e9. doi: 10.1016/j.stem.2020.10.016

Butler, A., Hoffman, P., Smibert, P., Papalexi, E., and Satija, R. (2018). Integrating single-cell transcriptomic data across different conditions, technologies, and species. Nat. Biotech. 36, 411-420. doi: 10.1038/nbt.4096

Caussinus, E., and Gonzale, C. (2005). Induction of tumor growth by altered stemcell asymmetric division in Drosophila melanogaster. Nat. Genet. 37, 1125-1129. doi: $10.1038 /$ ng 1632

Chaker, Z., Codega, P., and Doetsch, F. (2016). A mosaic world: puzzles revealed by adult neural stem cell heterogeneity. Wiley Interdiscip. Rev. Dev. Biol. 5, 640-658. doi: 10.1002/wdev.248 acquisition. All authors have read and agreed to the published version of the manuscript.

\section{FUNDING}

This study was supported by the funding from Associazione Italiana Ricerca sul Cancro (AIRC) to LC (IG 23052), from Regione Campania (PO FESR 2014-2020 SATIN) and from the European Union's Horizon 2020 Research and Innovation Programme VES4US (Grant agreement no. 801338) to GLL.

\section{ACKNOWLEDGMENTS}

We would like to acknowledge Antonio Simeone for the critical suggestions on in vivo BrdU-labeling, Sabrina Battista for the critical reading of the manuscript, Fabiana Franchini and Paola Francesca for the experimental contribution during their pregraduate internships, the IGB Integrated Microscopy Facility for support in image acquisition, and the IGB Animal Facility for mice housing.

\section{SUPPLEMENTARY MATERIAL}

The Supplementary Material for this article can be found online at: https://www.frontiersin.org/articles/10.3389/fcell.2021. 657149/full\#supplementary-material

Chiappetta, G., Valentino, T., Vitiello, M., Pasquinelli, R., Monaco, M., Palma, G., et al. (2015). PATZ1 acts as a tumor suppressor in thyroid cancer via targeting p53-dependent genes involved in EMT and cell migration. Oncotarget 6, 5310-5323. doi: 10.18632/oncotarget.2776

Cho, J. H., Kim, M. J., Kim, K. J., and Kim, J. R. (2012). POZ/BTB and AT-hookcontaining zinc finger protein 1 (PATZ1) inhibits endothelial cell senescence through a p53 dependent pathway. Cell Death Differ. 19, 703-712. doi: 10.1038/ cdd.2011.142

Christian, K. M., Song, H., and Ming, G. L. (2014). Functions and dysfunctions of adult hippocampal neurogenesis. Annu. Rev. Neurosci. 37, 243-262. doi: 10.1146/annurev-neuro-071013-014134

Cicalese, A., Bonizzi, G., Pasi, C. E., Faretta, M., Ronzoni, S., Giulini, B., et al. (2009). The tumor suppressor p53 regulates polarity of self-renewing divisions in mammary stem cells. Cell 138, 1083-1095. doi: 10.1016/j.cell.2009.06.048

Coles-Takabe, B. L., Brain, I., Purpura, K. A., Karpowicz, P., Zandstra, P. W., Morshead, C. M., et al. (2008). Don't look: growing clonal versus nonclonal neural stem cell colonies. Stem Cells 26, 2938-2944. doi: 10.1634/stemcells. 2008-0558

Costoya, J. A. (2007). Functional analysis of the role of POK transcriptional repressors. Brief. Funct. Genomic Proteomic 6, 8-18. doi: 10.1093/bfgp/elm002

Ellmeier, W. (2015). Molecular control of CD4(+) T cell lineage plasticity and integrity. Int. Immunopharmacol. 28, 813-817. doi: 10.1016/j.intimp.2015. 03.050

EMBL-EBI Expression Atlas (2020). Mouse RNA-Seq Time-Series of the Development of Seven Major Organs Experiment. Available online at: https://www.ebi.ac.uk/gxa/experiments/E-MTAB-6798/Downloads (accessed September 10, 2020).

Fedele, M., Benvenuto, G., Pero, R., Majello, B., Battista, S., Lembo, F., et al. (2000). A novel member of the BTB/POZ family, PATZ, associates with the RNF4 RING finger protein and acts as a transcriptional repressor. J. Biol. Chem. 275, 7894-7901. doi: 10.1074/jbc.275.11.7894 
Fedele, M., Cerchia, L., Pegoraro, S., Sgarra, R., and Manfioletti, G. (2019). Proneural-mesenchymal transition: phenotypic plasticity to acquire multitherapy resistance in glioblastoma. Int. J. Mol. Sci. 20:2746. doi: 10.3390/ ijms20112746

Fedele, M., Crescenzi, E., and Cerchia, L. (2017). The POZ/BTB and AThook containing zinc finger 1 (PATZ1) transcription regulator: physiological functions and disease involvement. Int. J. Mol. Sci. 18:2524. doi: 10.3390/ ijms 18122524

Fedele, M., Franco, R., Salvatore, G., Paronetto, M. P., Barbagallo, F., Pero, R., et al. (2008). PATZ1 gene has a critical role in the spermatogenesis and testicular tumours. J. Pathol. 215, 39-47. doi: 10.1002/path.2323

Fedele, M., Pierantoni, G. M., Pallante, P., and Fusco, A. (2012). High mobility group A-interacting proteins in cancer: focus on chromobox protein homolog 7, homeodomain interacting protein kinase 2 and PATZ. J. Nucl. Acids Inv. 3:e1. doi: 10.4081/jnai.2012.3988

Gaspard, N., Bouschet, T., Hourez, R., Dimidschstein, J., Naeije, G., van den Ameele, J., et al. (2008). An intrinsic mechanism of corticogenesis from embryonic stem cells. Nature 455, 351-357. doi: 10.1038/nature 07287

Giorgio, E., Liguoro, A., D’Orsi, L., Mancinelli, S., Barbieri, A., Palma, G., et al. (2014). Cripto haploinsufficiency affects in vivo colon tumor development. In. J. Oncol. 45, 31-40. doi: 10.3892/ijo.2014.2412

Guadagno, E., Vitiello, M., Francesca, P., Calì, G., Caponnetto, F., Cesselli, D., et al. (2017). PATZ1 is a new prognostic marker of glioblastoma associated with the stem-like phenotype and enriched in the proneural subtype. Oncotarget 8 , 59282-59300. doi: 10.18632/oncotarget. 19546

Hayes, N. L., and Nowakowski, R. S. (2002). Dynamics of cell proliferation in the adult dentate gyrus of two inbred strains of mice. Brain Res. Dev. Brain Res. 134, 77-85. doi: 10.1016/s0165-3806(01)00324-8

Hsieh, J., and Eisch, A. J. (2010). Epigenetics, hippocampal neurogenesis, and neuropsychiatric disorders: unraveling the genome to understand the mind. Neurobiol. Dis. 39, 73-84. doi: 10.1016/j.nbd.2010.01.008

Jabaudon, D. (2017). Fate and freedom in developing neocortical circuits. Nat. Commun. 8:16042. doi: 10.1038/ncomms16042

Jessberger, S., Clark, R. E., Broadbent, N. J., Clemenson, G. D. Jr., Consiglio, A., Lie, D. C., et al. (2009). Dentate gyrus-specific knockdown of adult neurogenesis impairs spatial and object recognition memory in adult rats. Learn. Mem. 16, 147-154. doi: 10.1101/lm.1172609

Kelly, K. F., and Daniel, J. M. (2006). POZ for effect-POZ-ZF transcription factors in cancer and development. Trends Cell Biol. 16, 578-587. doi: 10.1016/j.tcb. 2006.09.003

Keskin, N., Deniz, E., Eryilmaz, J., Un, M., Batur, T., Ersahin, T., et al. (2015). PATZ1 Is a DNA damage-responsive transcription factor that inhibits p53 function. Mol. Cell. Biol. 35, 1741-1753. doi: 10.1128/mcb.01475-14

Kuhn, H. G., Dickinson-Anson, H., and Gage, F. H. (1996). Neurogenesis in the dentate gyrus of the adult rat: age-related decrease of neuronal progenitor proliferation. J. Neurosci. 16, 2027-2033. doi: 10.1523/jneurosci.16-06-02027. 1996

Lee, J. H., Lee, J. E., Kahng, J. Y., Kim, S. H., Park, J. S., Yoon, S. J., et al. (2018). Human glioblastoma arises from subventricular zone cells with low-level driver mutations. Nature 560, 243-247. doi: 10.1038/s41586-018-0389-3

Liguori, G. L., Echevarria, D., Bonilla, S., D’Andrea, D., Liguoro, A., Persico, M. G., et al. (2009). Characterization of the functional properties of the neuroectoderm in mouse Cripto(-/-) embryos showing severe gastrulation defects. Int. J. Dev. Biol. 53, 549-557. doi: 10.1387/ijdb.082650gl

Livak, K. J., and Schmittgen, T. D. (2001). Analysis of relative gene expression data using real-time quantitative PCR and the 2(-Delta Delta C(T) method. Methods 25, 402-408. doi: 10.1006/meth.2001.1262

Ma, H., Ow, J. R., Tan, B. C., Goh, Z., Feng, B., Loh, Y. H., et al. (2014). The dosage of Patz1 modulates reprogramming process. Sci. Rep. 4:7519. doi: 10. 1038/srep07519

Mancinelli, S., and Lodato, S. (2018). Decoding neuronal diversity in the developing cerebral cortex: from single cells to functional networks. Curr. Opin. Neurobiol. 53, 146-155. doi: 10.1016/j.conb.2018.08.001

Monaco, M., Palma, G., Vitiello, M., Capiluongo, A., D'Andrea, B., Vuttariello, E., et al. (2018). Loss of one or Two PATZ1 Alleles has a critical role in the progression of thyroid carcinomas induced by the RET/PTC1 oncogene. Cancers (Basel) 10:92. doi: 10.3390/cancers10040092
Morshead, C. M., Garcia, A. D., Sofroniew, M. V., and van Der Kooy, D. (2003). The ablation of glial fibrillary acidic protein-positive cells from the adult central nervous system results in the loss of forebrain neural stem cells but not retinal stem cells. Eur. J. Neurosci. 18, 76-84. doi: 10.1046/j.1460-9568.2003.02727.x

Okamoto, M., Miyata, T., Konno, D., Ueda, H. R., Kasukawa, T., Hashimoto, M., et al. (2016). Cell-cycle-independent transitions in temporal identity of mammalian neural progenitor cells. Nat. Commun. 7:11349. doi: 10.1038/ ncomms 11349

Oliviero, G., Munawar, N., Watson, A., Streubel, G., Manning, G., Bardwell, V., et al. (2015). The variant polycomb repressor complex 1 component PCGF1 interacts with a pluripotency sub-network that includes DPPA4, a regulator of embryogenesis. Sci. Rep. 5:18388. doi: 10.1038/srep 18388

Orola, M. J., Tizian, C., Zhu, C., Andersen, L., Gülich, A. F., Alteneder, M., et al. (2019). The zinc-finger transcription factor MAZR regulates iNKT cell subset differentiation. Cell. Mol. Life Sci. 76, 4391-4404. doi: 10.1007/s00018-01903119-z

Ow, J. R., Ma, H., Jean, A., Goh, Z., Lee, Y. H., Chong, Y. M., et al. (2014). Patz1 regulates embryonic stem cell identity. Stem Cells Dev. 23, 1062-1073. doi: 10.1089/scd.2013.0430

Papatheodorou, I., Moreno, P., Manning, J., Fuentes, A. M., George, N., Fexova, S., et al. (2020). Expression atlas update: from tissues to single cells. Nucl. Acids Res. 48, D77-D83.

Passariello, A., Errico, M. E., Donofrio, V., Maestrini, M., Zerbato, A., Cerchia, L., et al. (2019). PATZ1 is overexpressed in pediatric glial tumors and correlates with worse event-free survival in high-grade gliomas. Cancers (Basel) 11:1537. doi: 10.3390/cancers 11101537

Pisapia, L., Terreri, S., Barba, P., Mastroianni, M., Donnini, M., Mercadante, V., et al. (2020). Role of PA2G4P4 pseudogene in bladder cancer tumorigenesis. Biology (Basel) 9:66. doi: 10.3390/biology9040066

Puca, F., Tosti, N., Federico, A., Kuzay, Y., Pepe, A., Morlando, S., et al. (2019). HMGA1 negatively regulates NUMB expression at transcriptional and post transcriptional level in glioblastoma stem cells. Cell Cycle 18, 1446-1457. doi: $10.1080 / 15384101.2019 .1618541$

Q4Lab (2016). Protocol - Fixation and Paraffin Embedding of Tissue Samples. Available online at: http:/quality4lab.igb.cnr.it/en/protocols/histology/fixingthe-embryos-and-wax-embedding (accessed August 10, 2020).

Rueden, C. T., Schindelin, J., Hiner, M. C., DeZonia, B. E., Walter, A. E., Arena, E. T., et al. (2017). ImageJ2: imageJ for the next generation of scientific image data. BMC Bioinformatics 18:529. doi: 10.1186/s12859-0171934-z

Sahay, A., Scobie, K. N., Hill, A. S., O'Carroll, C. M., Kheirbek, M. A., Burghardt, N. S., et al. (2011). Increasing adult hippocampal neurogenesis is sufficient to improve pattern separation. Nature 472, 466-470. doi: 10.1038/nature 09817

Sakaguchi, S., Hombauer, M., Bilic, I., Naoe, Y., Schebesta, A., Taniuchi, I., et al. (2010). The zinc-fingerprotein MAZR is part of the transcription factor network that controls the CD4 versus CD8 lineage fate of double-positive thymocytes. Nat. Immunol. 11, 442-448. doi: 10.1038/ni.1860

Satija, R., Farrell, J. A., Gennert, D., Schier, A. F., and Regev, A. (2015). Spatial reconstruction of single-cell gene expression data. Nat. Biotech. 33, 495-502. doi: $10.1038 /$ nbt. 3192

Schilder, B. M., and Raj, T. (2020). Fine-mapping of Parkinson's disease susceptibility loci identifies putative causal variants. bioRxiv [Preprint]. doi: $10.1101 / 2020.10 .22 .340158$

Scopa, C., Marrocco, F., Latina, V., Ruggeri, F., Corvaglia, V., La Regina, F., et al. (2020). Impaired adult neurogenesis is an early event in Alzheimer' disease neurodegeneration, mediated by intracellular Abeta oligomers. Cell Death Differ. 27, 934-948. doi: 10.1038/s41418-019-0409-3

Stuart, T., Butler, A., Hoffman, P., Hafemeister, C., Papalexi, E., Mauck, W. M. III, et al. (2019). Comprehensive integration of single-cell data. Cell 177, 1888-1902. doi: $10.1016 /$ j.cell.2019.05.031

Sturm, D., Bender, S., Jones, D. T., Lichter, P., Grill, J., Becher, O., et al. (2014). Paediatric and adult glioblastoma: multiform (epi)genomic culprits emerge. Nat. Rev. Cancer 14, 92-107. doi: 10.1038/nrc3655

Telley, L., Agirman, G., Prados, J., Amberg, N., Fièvre, S., Oberst, P., et al. (2019). Temporal patterning of apical progenitors and their daughter neurons in the developing neocortex. Science 364:eaav2522. doi: 10.1126/science.aav2522 
Valentino, T., Palmieri, D., Vitiello, M., Pierantoni, G. M., Fusco, A., and Fedele, M. (2013a). PATZ1 interacts with p53 and regulates expression of p53-target genes enhancing apoptosis or cell survival based on the cellular context. Cell Death Dis. 4:e963. doi: 10.1038/cddis.2013. 500

Valentino, T., Palmieri, D., Vitiello, M., Simeone, A., Palma, G., Arra, C., et al. (2013b). Embryonic defects and growth alteration in mice with homozygous disruption of the Patzl gene. J. Cell. Physiol. 228, 646-653. doi: 10.1002/jcp. 24174

Vitiello, M., Palma, G., Monaco, M., Bello, A. M., Camorani, S., Francesca, P., et al. (2019). Dual oncogenic / anti-oncogenic role of PATZ1 in FRTL5 rat thyroid cells transformed by the Ha-Ras V12 oncogene. Genes (Basel) 10:127. doi: 10.3390/genes10020127

Xu, F., Yang, J., Chen, J., Wu, Q., Gong, W., Zhang, J., et al. (2015). Differential co-expression and regulation analyses reveal different mechanisms underlying major depressive disorder and subsyndromal symptomatic depression. BMC Bioinformatics 16:112. doi: 10.1186/s12859-0150543-y

Xu, W., Yao, X., Zhao, F., Zhao, H., Cheng, Z., Yang, W., et al. (2020). Changes in hippocampal plasticity in depression and therapeutic approaches influencing these changes. Neural. Plast. 2020:8861903. doi: 10.1155/2020/88 61903

Yao, B., Christian, K. M., He, C., Jin, P., Ming, G. L., and Song, H. (2016). Epigenetic mechanisms in neurogenesis. Nat. Rev. Neurosci. 17, 537-549. doi: 10.1038/nrn. 2016.70

Zhang, J., and Jiao, J. (2015). Molecular biomarkers for embryonic and adult neural stem cells and neurogenesis. Biomed. Res. Int. 2015:727542. doi: 10.1155/2015/ 727542

Conflict of Interest: The authors declare that the research was conducted in the absence of any commercial or financial relationships that could be construed as a potential conflict of interest.

Copyright (C) 2021 Mancinelli, Vitiello, Donnini, Mantile, Palma, Luciano, Arra, Cerchia, Liguori and Fedele. This is an open-access article distributed under the terms of the Creative Commons Attribution License (CC BY). The use, distribution or reproduction in other forums is permitted, provided the original author(s) and the copyright owner(s) are credited and that the original publication in this journal is cited, in accordance with accepted academic practice. No use, distribution or reproduction is permitted which does not comply with these terms. 